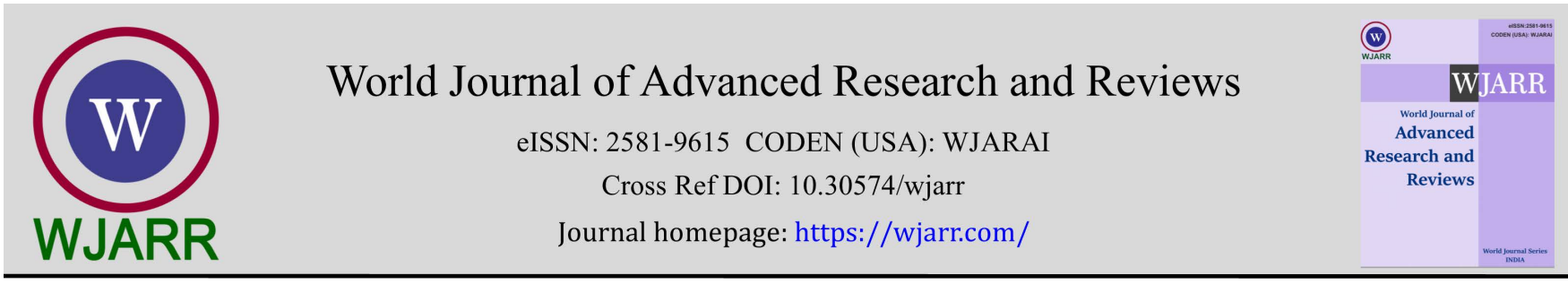

(CASE REPORT)

\title{
Acute mesenteric ischemia in SARS CoV 2: A conventional approach
}

\author{
Arushi Mohan *, Abhinaya Shekhar, Brunda MS, Chaithanya Soma Chetty \\ Internal medicine, Aster CMI hospital Bengaluru, Karnataka, India.
}

World Journal of Advanced Research and Reviews, 2021, 11(01), 008-012

Publication history: Received on 24 May 2021; revised on 26 June 2021; accepted on 29 June 2021

Article DOI: https://doi.org/10.30574/wjarr.2021.11.1.0299

\begin{abstract}
SARS CoV 2, a novel coronavirus, an airborne virus has been investigated for its inconspicuous prothrombotic states, the mesenteric vessels are one of the many devoured by the virus. Gastrointestinal symptoms may range from a mild gastroenteritis to a grave ischemia indicating a possibility of a severe infection. The hypercoagulability in SARS Cov -2 is possibly related to the hypercoagulable state attributed by the heightened systemic inflammatory response. The interplay of anticoagulants and the immune response is crucial in the management of critical patients , D dimer a fibrin degradation product has demonstrated efficacy in the surveille of coagulability.This case study involved in the scrutiny of a case with the initial presentation of symptoms of lower respiratory tract infection progressing into gastrointestinal symptoms focusses on the medical management of acute mesenteric ischemia in SARS CoV 2, stressing on the importance of early detection and prompt management of the hypercoagulable condition in systems other than the pulmonary system associated with SARS CoV 2. A meticulous analysis of ischemic states in various other organs should be sought in the pandemic.
\end{abstract}

Keywords: Acute mesenteric ischemia; SARS CoV 2; Case study; Hypercoagulability; D dimer; Gastrointestinal

\section{Introduction}

The high number of arterial and, in particular, venous thromboembolic events diagnosed within $24 \mathrm{~h}$ of admission and the high rate of positive VTE (venous thromboembolism) imaging tests among the few COVID-19 patients tested suggest that there is an urgent need to improve specific VTE diagnostic strategies and investigate the efficacy and safety of thromboprophylaxis in ambulatory COVID-19 patients [1].

Within severe cases of patients with COVID-19, the incidence of a symptomatology or gastro-intestinal complications is high (39-73.8\%).

COVID-19 may predispose to both venous and arterial thromboembolism due to Excessive inflammation, hypoxia, and immobilization and diffuse intravascular coagulation [2].

\section{Discussion}

The exact pathological mechanism underpinning the complication of acute mesenteric ischemia in COVID-19 is not known at present. Putatively, four mechanisms, in isolation or in varying combinations could account for this fulminant complication in severe COVID-19 cases. First, hypercoagulability is a product of a well-known phenomenon called "cytokine storm" a systemic inflammatory state, product of the over-activation of the immune system against the virus. Endothelial activation is a process where endothelial cells expose receptors in their membranes and attract monocytes in order to perpetuate the cytokine synthesis and liberation resulting in the induction of vasodilatation (with loss of

\footnotetext{
* Corresponding author: Arushi Mohan

Internal medicine, Aster CMI hospital Bengaluru, Karnataka, India.

Copyright $(2021$ Author(s) retain the copyright of this article. This article is published under the terms of the Creative Commons Attribution Liscense 4.0.
} 
plasma making the blood even more viscose). Hypoxia is a common feature in COVID-19 patients. Lung's inflammation caused by the virus makes the hemostasis very difficult to carry on without implementation of mechanics respiratory assistance. COVID-19 patients who need to be in the ICU suffered a very long stay in there. An average of 17-23 days is the regular stay in the intensive care units that is why immobilization is another factor that makes this hypercoagulability more intense.

A high level of suspicion for intestinal ischemia should be maintained in COVID-19 patients presenting with GI (Gastrointestinal) symptoms or with arising abdominal pain as this complication could account for an increased mortality risk. Nevertheless, in this subset, a D-dimer elevation should not only trigger prompt prophylactic use of anticoagulants, but also lead to consideration of an early abdominal CT (computed tomography) scan in patients with suggestive symptoms or biochemical markers of intestinal ischemia. Additional analysis will help to define the role of SARS-CoV-2 in the pathogenesis of such a detrimental manifestation [3].

An unexplained clinical picture should raise the suspicion for rare conditions such as mesenteric thrombosis. Adequate prophylactic measures should be implemented both during hospitalization and after discharge [4].

Patients with COVID-19 disease should be managed with a holistic approach with evaluation of digestive and respiratory symptoms. In all patients with COVID-19, routine anticoagulation with heparin with regular monitoring of coagulation tests may prevent thrombotic complications. Mesenteric ischemia should be suspected if the patient has abdominal distension or pain with increased inflammatory markers. Contrast-enhanced CT scan should be done for diagnosis and may be repeated to see the progression of disease. Early intervention is lifesaving [5].

In conclusion, these findings advocate for a reactive approach with early abdominal CT scans in patients with unexplained worsening status during COVID-19. Exploratory laparotomy and potentially bowel resection should be further considered, if signs of small bowel involvement are detected [6].

\section{Case report}

A 55-year female COVID positive by RT PCR was admitted to the hospital in view of gastrointestinal symptoms with complaints of abdominal pain for 2 days which was acute in nature, progressive and diffuse. No history of radiation of pain. Patient complains of loose stools for 1 day 2-3 episodes watery stool (not blood tinged, no mucous). No association with nausea/vomiting. No significant family history, No history of travel, no history of change in diet. Past history: Known case of type-2 diabetes mellitus for 6 months.

\section{Physical Examination}

On admission: Vitals stable with saturation @ 97\% on room air.

Random blood glucose was found to be elevated Glasgow coma scale 15/15.

Pupils equal round and reactive, all peripheral pulses felt, no history of allergies.

Arterial blood gas on admission showed the presence of metabolic acidosis and a lactic acid level of $4 \mathrm{mmol} / \mathrm{litre}$.

Abdominal examination: Shows soft abdomen, diffuse tenderness more in the right lower lumbar region.

\section{Relevant laboratory and Radiological Investigations}

On Admission:

D dimer: $6535.04 \mathrm{ng} / \mathrm{ml}($ Normal: <500)

PT (prothrombin time): 94seconds (Normal: 11-13.5)

INR (international normalized ratio): 1.51(Normal: 0.8-1.1)

Activated partial thromboplastin time (aPTT): 50.1seconds (Normal: 30-40 seconds)

Serum creatinine, uric acid, electrolytes, liver function, lactate dehydrogenase, ferritin were found to be within normal limits. Haemoglobin was low.

Imaging studies: CT (computed tomography) abdomen and Pelvis done on the day of admission which showed diffuse thrombosis involving portal vein, splenic vein and superior mesenteric vein. The thrombus extends into right and left portal vein branches. Diffuse edematous wall thickening of few small bowel loops with one of the proximal jejunal loops 
which was relatively non-enhancing- suggestive of an ischemic small bowel with mild ascites. Both lungs show typical findings of COVID-19 pneumonia. CT severity score for COVID-19 is 19/25.

A repeat $\mathrm{CT}$ abdomen and pelvis done on the third day of admission showed: there is partial recanalization of superior mesenteric vein, the bowel loops are hypo enhancing. There is mild interval reduction in the edema wall and thickening of the bowel loops. Surrounding mesenteric fat stranding was found. Mild to moderate ascites noted. Interval appearance of bilateral pleural effusion $(R>L)$ - Moderate to gross pleural effusion on right side. There is partial compressive atelectasis of right lung. Multifocal as well as diffuse areas of ground glass opacities, focal consolidations with reticular interlobular and intralobular interstitial thickening are noted in bilateral lung parenchyma involving all lobes.

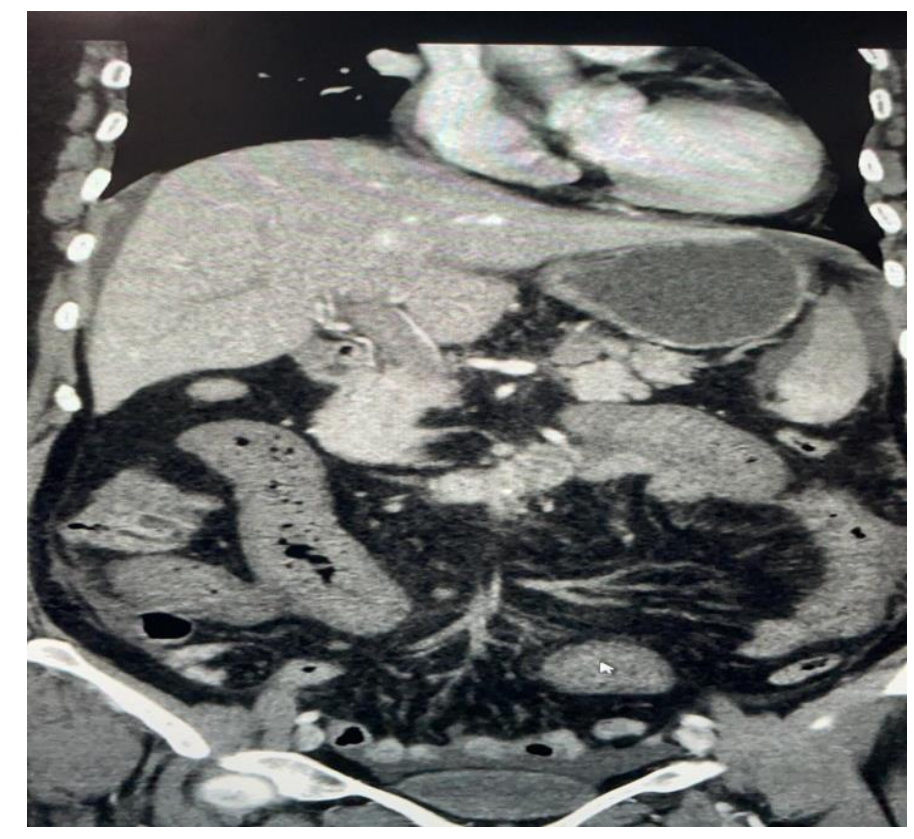

Figure 1 CT Abdomen and pelvis (Longitudinal view)

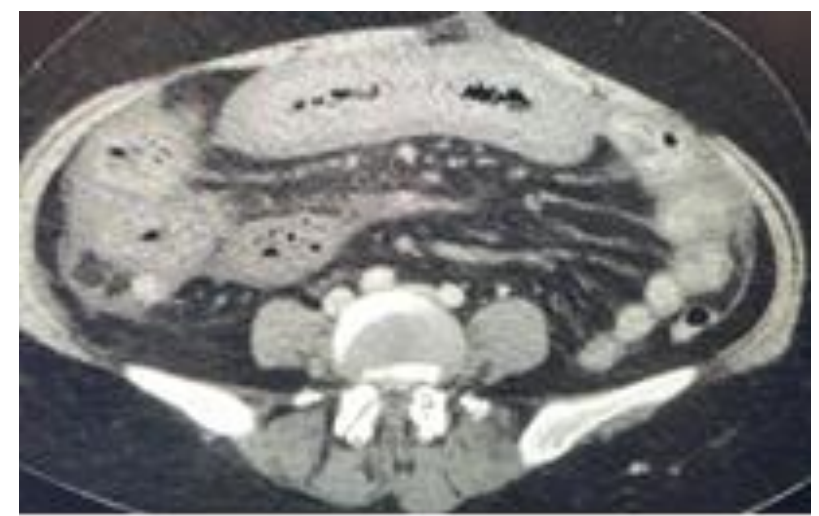

Figure 2 CT abdomen and pelvis (Cross sectional view)

Pulmonary: A week after admission when ultrasound of the chest was done due to excessive dyspnea findings of a massive pleural effusion on the right side were seen for which pleural tap under aseptic precautions was done which revealed $700 \mathrm{ml}$ of fluid with the following analysis.

Total count: 134 cells /mm3. Differential count neutrophils: 5\% Lymphocytes: 95\%. Acid fast bacilli: negative Culture sensitivity: on aerobic method of culture showed no growth. Bronchoalveolar lavage: showed the presence of inflammatory cells. 
Anticoagulation: patient underwent anticoagulation, alerting surgeons in case of an emergent laparoscopic procedure. Patient was started on Heparin (IV) 5000units /hour infusion given for 4 days (anticoagulants were withheld) followed by a low molecular weight heparin (enoxaparin $40 \mathrm{mg} \mathrm{S} / \mathrm{c}$ twice a day dose with daily monitoring twice a day of her Aptt and hemoglobin) for 4 days from admission in view of elevated D dimer levels. Patient's PTT varied b/w $48.8--50.2$ and hemoglobin varied between 7.6 and 10 .

Antiviral and antibiotic: Patient were started on Remdesivir on the second day of admission due to a CT score of 19/25. Patient was also started on Vitamin C 500mg on zinc supplements and Antibiotic (IV) meropenem $1 \mathrm{gm}$ given for 12 days individualized escalation of antibiotics was performed.

Pain: Patient was initially placed on Acetaminophen 1 gm (IV) was given SOS but was given 4 times a day for 4 days from admission followed by Tramadol 50mg (IV) thrice a day in view of abdominal pain.

Oxygen requirement: Due to a drop in saturation 02 was started @ 6 liters/min patient initially required 6 liters followed by fluctuations in the saturation which increased to 10 liters 02 and post pleural tap patient was weaned off 02. Budesonide nebulization + furosemide- $40 \mathrm{mg}$ for 4 days, twice a day was started in view of respiratory distress.

Nutritional management: When the patient presented with abdominal pain, she was advised nil per oral and started on intravenous fluids followed by insertion of a central line for providing parenteral nutrition at $30 \mathrm{ml} / \mathrm{hr}$. following removal of nasogastric tube. Albumin 20\% OD was also given. A phosphorus sachet twice a day was added in view of low phosphorus levels.

Chronic diseases: For type-2 diabetes mellitus patient was on oral hypoglycemic agents: (metformin + glimepiride). Insulin as per sliding scale was given in the hospital. Patient was also started on a calcium channel blocker Amlodipine $5 \mathrm{mg}$ SOS if BP got greater than 140/90.

Patient was discharged in a stable condition.

\section{Conclusion}

In SARS CoV-2 positive patients with elevated inflammatory markers, a possibility of other organ involvements should be considered.

Early initiation of anticoagulation in patients with COVID-19 can result in reduced risk of thromboembolic complications.

\section{Compliance with ethical standards}

\section{Acknowledgments}

We would like to thank Kinshuk Kalia for helping with formatting the document. Facundo Garcia Barberá City-Country: Córdoba- Argentina National University of Córdoba.for editing and reviewing the document.

\section{Disclosure of conflict of interest}

No conflict of interest.

\section{Statement of informed consent}

Informed consent was obtained from all individual participants included in the study.

\section{References}

[1] Cheung S, Quiwa JC, Pillai A, Onwu C, Tharayil ZJ, Gupta R. Superior mesenteric artery thrombosis and acute intestinal ischemia as a consequence of COVID-19 infection. The American Journal of Case Reports. 2020;2: e925753-1.

[2] a Beccara L, Pacioni C, Ponton S, Francavilla S, Cuzzoli A. Arterial mesenteric thrombosis as a complication of SARS-CoV-2 infection. European journal of case reports in internal medicine. 2020;7(5). 
[3] Norsa L, Bonaffini PA, Indriolo A, Valle C, Sonzogni A, Sironi S. Poor outcome of intestinal ischemic manifestations of COVID-19. Gastroenterology. 2020 Oct 1;159(4):1595-7.

[4] Rodriguez-Nakamura RM, Gonzalez-Calatayud M, Martinez AR. Acute mesenteric thrombosis in two patients with COVID-19. Two cases report and literature review. International Journal of Surgery Case Reports. 2020 Oct 15.

[5] Karna ST, Panda R, Maurya AP, Kumari S. Superior mesenteric artery thrombosis in COVID-19 pneumonia: an underestimated diagnosis—first case report in Asia. Indian Journal of Surgery. 2020 Dec;82(6):1235-7.

[6] Ignat M, Philouze G, Aussenac-Belle L, Faucher V, Collange O, Mutter D, Pessaux P. Small bowel ischemia and SARS-CoV-2 infection: an underdiagnosed distinct clinical entity. Surgery. 2020 Jul 1;168(1):14-6. 